\title{
PHOIBOS: probing heliospheric origins with an inner boundary observing spacecraft
}

\author{
M. Maksimovic $\cdot$ M. Velli
}

Received: 30 November 2007 / Accepted: 21 March 2008 / Published online: 16 August 2008

(C) Springer Science + Business Media B.V. 2008

\begin{abstract}
The earth is immersed in a hot, rarefied, energetic flow of particles and electromagnetic fields originating from the Sun and engulfing the entire solar system, forming the heliosphere. The existence of the solar wind has been established for almost 50 years now, and abundant data has been accumulated concerning both its average properties and the intermittent, violent energetic manifestations known as Coronal Mass Ejections which often impact the earth's magnetosphere (causing geomagnetic storms and aurorae). The mystery of how the solar corona is heated and the solar wind is accelerated remains unsolved, however, because of the large gap in our knowledge of the inner region of the heliosphere, inside the orbit of mercury. The PHOIBOS mission, with a perihelion at $4 \mathrm{R}_{\mathrm{s}}$, by accessing the regions where energy in the coronal plasma is channeled from internal, magnetic and turbulent energy into bulk energy of the solar wind flow aims to solve the question of why the Sun has a hot corona and produces a solar wind. The PHOIBOS mission builds on previous Solar Probe studies, but provides an alternative orbit scenario avoiding a Jupiter encounter in favor of multiple Venus encounters and SEP systems to work its way close to the Sun in a gradual manner, providing a much vaster data return.
\end{abstract}

Keywords Solar corona $\cdot$ Solar wind acceleration $\cdot$ Coronal heating $\cdot$ Space mission

\footnotetext{
M. Maksimovic $(\triangle)$

LESIA, Observatoire de Paris, CNRS, UPMC, Universite Paris Diderot, 92195 Meudon cedex, France

e-mail: milan.maksimovic@obspm.fr

M. Velli

Jet Propulsion Laboratory, 4800 Oak Grove Drive, Pasadena 91109, CA, USA

M. Velli

Università degli Studi, Florence, Italy
} 


\section{Introduction}

Since the first realization in the 1940s that the solar corona has a temperature of a few million degrees, and that therefore the outer solar atmosphere can not be in equilibrium but must expand supersonically (as predicted by Parker in the late 1950s), to create the Heliosphere - a solar controlled cavity in the interplanetary medium - many advances have occurred in our understanding of the Sun and its influence on the solar system through experiments in space: the Helios spacecraft have observed the region between 0.3 and $1 \mathrm{AU}$ in the ecliptic plane, the Voyager spacecraft have reached the outer confines of the Heliosphere, and the Ulysses mission has completed out of ecliptic orbits between 1.5 and 5 AU giving us a threedimensional view of the solar wind. The composition, properties and structure of the solar wind have been extensively measured, and its dependence on solar cycle and helio-magnetic latitude have been charted. Remote sensing experiments in space have given us high-resolution views of the corona, transition region and photosphere, revealing the complex and dynamic structuring of the atmosphere by magnetic fields.

Observational and experimental advances have been accompanied by progress in theories and modeling, which are now capable of giving a broad macroscopic phenomenological description of the major features of the observed solar wind outflow. Yet the fundamental questions of why the solar corona is so much hotter than the visible photosphere, and how and in what form this hot plasma than escapes from the Sun to give rise to the solar wind, remain basically unanswered. The magnetic field generated by the solar dynamo seems to play a major role both in defining the structure of the coronal and solar wind outflow as well as in channeling the energy from the outer layers of the Sun. Yet our knowledge of the magnetic field dynamics in the corona and nascent solar wind is sparse.

After decades of observational and theoretical studies, it has become apparent that definite answers to these questions can be obtained only through in-situ measurements of the solar wind down in the corona. The reason is that we observe the solar wind as a supersonic, continuous outflow, in a region where it is completely dominated by the outflow energy, but to really understand the acceleration and thermodynamics of the acceleration we need measurements inside the radius where the magnetic and thermal energies are still a dominant component. Extended in-situ measurements within this region, inside around $20 \mathrm{R}_{\mathrm{s}}$ from the Sun, and correlation of plasma measurements with solar source regions, will unlock the secrets of coronal heating and expansion. To date, the closest any spacecraft (Helios 1 and 2) have come to the Sun is $65 \mathrm{R}_{\mathrm{s}}$, far outside the region where acceleration occurs. Thus a mission to explore the solar corona is as timely today, or even more so, than at the time the first studies were carried out 30 years ago. Both NASA and ESA developed scenarios for a Solar Probe (Fig. 1). The PHOIBOS scenario presented here differs mainly on two points. Firstly, a classical solar panels technology combined with heat shield thermal generators are used as power systems instead of Radioisotope Thermoelectric Generators (RTGs). This is because RTGs are not currently available in Europe. Secondly, a new original trajectory combined with the use of a plasmic electric propulsion system are suggested in order to reach the final orbit instead of the classical Jupiter low altitude swing-by with a very high escape energy from 
Fig. 1 Artist's concept of PHOIBOS approaching the Sun

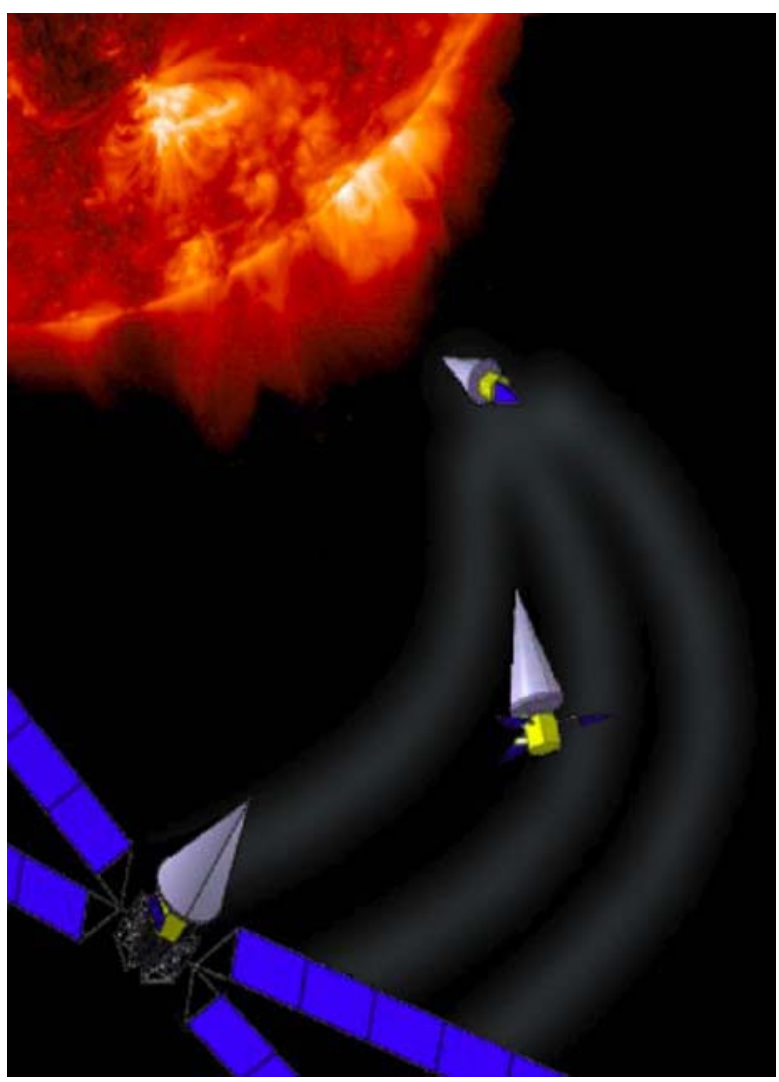

Earth. The PHOIBOS mission profile allows therefore more flexibility in the fine adjustment of the final orbit. It may avoid the possible drawbacks of a Jupiter swingby such as the high departure $V \infty$, the risky low altitude Jupiter swing-by at $5.5 \mathrm{AU}$ with a significant radiation level and no consequent trajectory changing capability [17].

The primary scientific goal of PHOIBOS will be to determine the structure and dynamics of plasmas and magnetic fields in the outer solar atmosphere which give rise to the corona, the solar wind and the heliosphere throughout the Sun's activity cycle. With a perihelion well within the region where the magnetic field still guides the plasma outflow and the solar wind kinetic energy is dominated by that of the Sun's magnetic field - the Alfvén critical point, somewhere between 10 and $20 R_{s}$ PHOIBOS will be able to access the regions of the corona where the plasma turbulence could be the strongest, most probably reaching, at perihelion, the location of the proton temperature maximum; it will travel across coronal magnetic structures in regions where field-aligned plasma flows have yet to interact dynamically and mix in the wind, and will be able to image the source regions of the plasma and magnetic structures it is crossing.

Subsequent sections of this paper summarize the scientific objectives and science implementation for the PHOIBOS mission as defined in the recent proposal 
submitted in response to the ESA Cosmic Visions call [15]. Further insight into Solar Probe studies and the necessity for such a mission to understand the origin of the heliosphere may be found in the 2005 NASA Science and Technology definition team report [17].

\section{Solar probe mission science objectives}

The solar wind flow at solar minimum is structured into high and low speed streams, with speeds of around 750 and $400 \mathrm{~km} / \mathrm{s}$ respectively. The Ulysses mission has shown that the fast wind is the basic outflow from the corona at solar minimum, while the much more irregular slow solar wind is confined to the equatorial regions, presumably arising from regions adjacent or inside the streamer belt. As the solar cycle progresses, the streamer belt expands in latitude so that, at activity maximum, the corona appears to be nearly uniformly distributed around the solar disk, and high speed wind streams occur over a much smaller volume, as illustrated by the 'dial plot' in Fig. 2, [16] which depicts solar wind speed measurements as a function of latitude during the first and second Ulysses orbits, near times of solar minimum and maximum activity respectively.

The fast solar wind, with a speed around $750 \mathrm{~km} / \mathrm{s}$, originates from coronal holes where the coronal electron temperature is cooler [3]. This inverse correlation between flow speed and coronal electron temperature (where the freezing in of minor ion charge states occurs) shows that one of the basic tenets of the original theory of the solar wind, which is based on high coronal electron temperatures and heat conduction, needs to be reconsidered. SOHO measurements of the very high temperatures of the coronal ions $[12,13]$, together with the persistent positive correlation of in-situ wind speed and proton temperature, suggest that other forces,
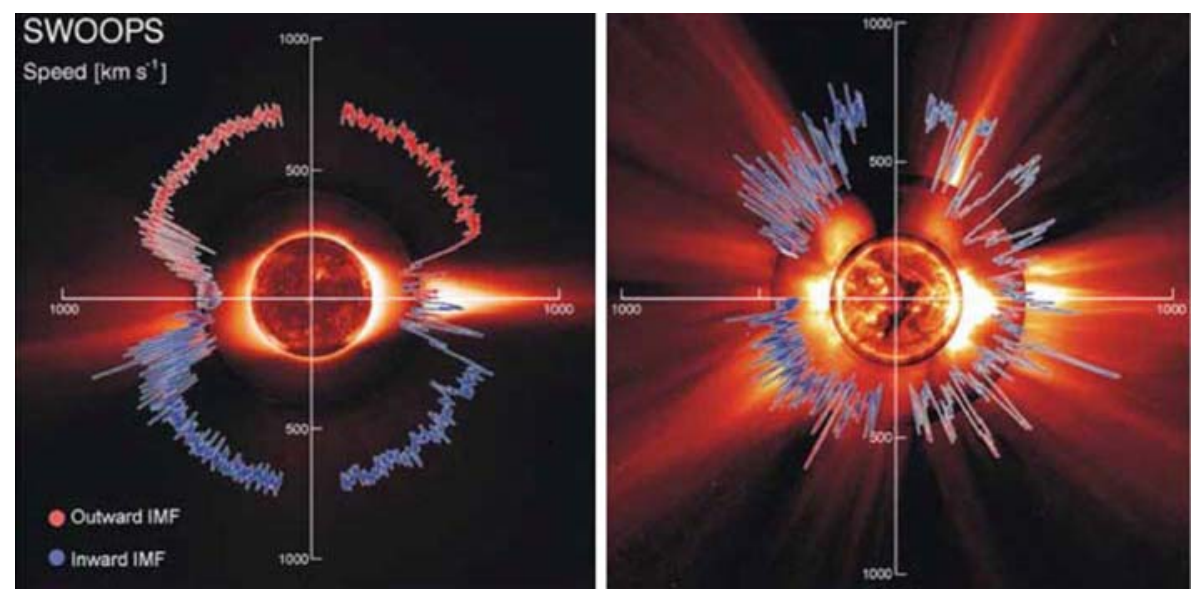

Fig. 2 The solar wind at solar minimum has a simple bimodal structure: high speed wind from polar coronal holes, low speed wind from the equatorial current sheet. At solar maximum, this gives way to a much more complex superposition of high and low speed jets [16] 
namely magnetic mirror and wave-particle interactions should also contribute strongly to the expansion of the outer corona. SOHO observations have also made important contributions to our knowledge of the slow solar wind, which is confined to regions emanating from the magnetic activity belt and seems to expand in a bursty, intermittent fashion from the top of helmet streamers, seen to expand continuously, in X-rays, by Yohkoh. A third type of flow arises from larger eruptions of coronal magnetic structures, or coronal mass ejections (CMEs), which also lead to acceleration of high-energy particles. As the solar activity cycle progresses, Ulysses has shown how the simple bi-modal structure gives way to a much more variable, but typically slower, solar wind at activity maximum, apparently originating not only from the much more sparse coronal hole regions and quiet Sun, but also from coronal active regions.

The energy that heats the corona and drives the wind is believed to come from photospheric motions and is channeled, stored and dissipated by the magnetic fields that emerge from the photosphere and structure the coronal plasma.

Several fundamental plasma physical processes - waves and instabilities, magnetic reconnection, velocity filtration, turbulent cascades - operating on a vast range of temporal and spatial scales are believed to play a role in coronal heating and solar wind acceleration, but the lack of magnetic field and detailed plasma measurements in the region inside $70 \mathrm{R}_{\mathrm{s}}$ does not allow their validation or confutation at this time, and though Solar Orbiter and Solar Sentinels will move inside 0.25 AU or $53 R_{s}$ before 2020, only PHOIBOS will explore the critical regions within $20 R_{s}$.

Basic unanswered questions concern the storage, transport, and release of the mechanical energy required for coronal heating; the specific mechanism(s) for the conversion of energy between the magnetic field and thermal particles; the dynamics of photospheric and coronal magnetic fields in the source regions of the solar wind; and the sources of high-energy particles and the mechanisms by which they are accelerated.

These questions motivate three broadly distinct but interlinked top-level PHOIBOS objectives. A fourth top-level objective of an exploratory nature concerns the source, composition, and dynamics of dust in the inner solar system.

\footnotetext{
PHOIBOS scientific objectives

1-Explore the fundamental processes underlying coronal heating and solar wind acceleration

2-Determine magnetic field structure and dynamics in the source regions of the fast and slow solar wind.

3-What mechanisms accelerate, store, and transport energetic particles?

4-Explore dust and dusty plasma phenomena and their influence on the solar wind and energetic particle formation.
}

2.1 Explore the fundamental processes underlying coronal heating and solar wind acceleration

Remote-sensing measurements of the solar corona and in-situ measurement of particle distribution functions in the fast and slow solar wind streams have shown that the coronal heating process is correlated with magnetic structure. SOHO/UVCS observations using the Doppler dimming technique [12, 13] (Fig. 3) and interplanetary scintillation measurements [9] indicate that the high speed solar wind 


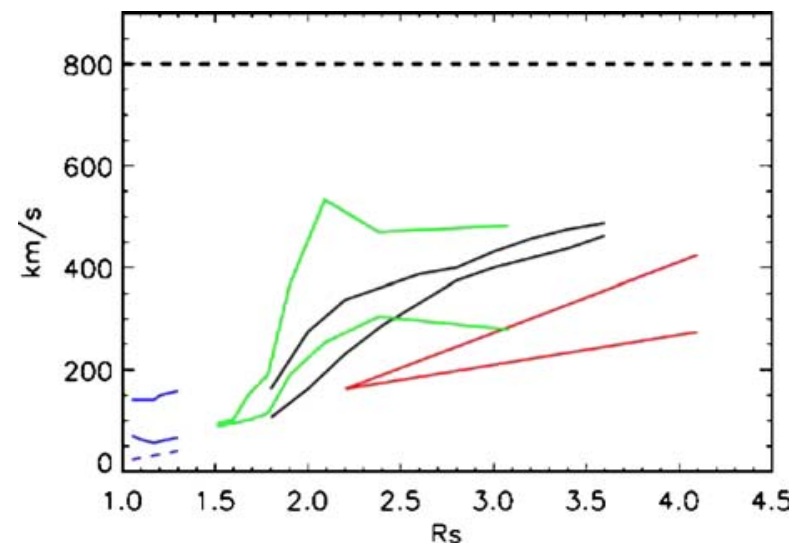

Fig. 3 Acceleration profiles of the fast solar wind after SoHO: $\mathrm{H}^{0}\left(\right.$ red) and $\mathrm{O}^{+5}$ (black) flow velocities from [12], $\mathrm{O}^{\mathrm{VI}}$ (green) flow velocities from [1]; full-dashed blue lines : plume and interplume flow velocities from [7]

is rapidly accelerated near the Sun, reaching speeds of the order of $600 \mathrm{~km} / \mathrm{s}$ within $10 \mathrm{R}_{\mathrm{s}}$. Such rapid acceleration appears to result from the extremely large and anisotropic effective temperatures in the lower corona, which have been measured by $\mathrm{SOHO} / \mathrm{UVCS}$ in coronal holes, though not directly for protons, the main solar wind constituent. These temperatures are much higher perpendicular to the magnetic field. The fast solar wind measured in situ shows what may be a relic of this anisotropy, smaller than that inferred from coronal observations, but persisting in the distance range from 0.3 to 5 AU. Proton, alpha-particle, and minor ion distribution functions in the fast wind also present a non-thermal beam-like component whose speed is comparable to the local Alfvén speed. All these properties suggest that Alfvén or ion-cyclotron waves play a major role in coronal heating and solar wind acceleration in high-speed wind. Measurements close to the Sun, within the region where the solar wind becomes supersonic to Alfvén waves, are necessary to remove ambiguity due to in situ evolution and obtain direct measurements where the main acceleration is occurring. Models on acceleration of fast solar wind by MHD waves were recently reviewed by Ofman [20].

The fast solar wind flow is steady, with fluctuations in radial speed of order 50 $\mathrm{km} / \mathrm{s}$, and the charge-state distributions indicate a low freezing-in temperature. The slow solar wind is variable, with higher but variable freezing-in temperatures. The composition of the fast and slow wind also differs, $\mathrm{Mg}$ and Fe being overabundant with respect to $\mathrm{O}$ in the slow wind. Solar wind protons and ions are however typically hotter in high speed streams than in the slow wind. The difference between the fast and the slow solar wind extends to the shape of the particle distribution functions. The fast wind exhibits proton perpendicular temperatures which are slightly higher than the parallel temperatures. Proton distribution functions in the fast wind also present a beam accelerated compared to the main distribution by a speed comparable to the Alfvén speed, a feature shared by the alpha particles. Turbulence is also different in fast and slow streams, with fast streams containing stronger fluctuations in transverse velocity and magnetic fields which are correlated in what is known as Alfvénic turbulence, a well-developed spectrum of quasi-incompressible 
waves propagating away from the Sun. In the slow wind no such preferred sense of propagation is observed, while larger density and magnetic field magnitude fluctuations are present, revealing a much more standard and evolved MHD turbulent state [10].

Anti-correlation of wind speed with electron temperature and the heliospheric distribution of the high speed wind at solar minimum place the origin of the fast wind in coronal holes. Measurement from the CDS-SUMER experiments aboard SOHO have ascertained that the electron temperature is bounded by $10^{6} \mathrm{~K}$ [3], in agreement with the brightness temperature based on radio observations of the corona.

Contrary to proton distributions, observed electron velocity distribution functions (eVDFs) exhibit non-Maxwellian features whatever the type of wind, slow or fast, in which they are observed. The eVDFs permanently exhibit three different components: a thermal core and a supra-thermal halo, which are always present at all pitch angles, and a sharply magnetic field aligned "strahl" which is usually antisunward-moving [22]. Energy transport and dissipation mechanisms strongly depend on the mean free path of particles in the coronal plasma, which varies drastically both with distance from the Sun (from the base of the corona to the supersonic solar wind), as well as across coronal structures (coronal holes to helmet streamers). This dependence has led to the suggestion that coronal heating arises from energy stored in non-thermal wings of particle distribution functions generated between the chromosphere and transition region or, more generally, in the region where the solar atmospheric plasma changes from collisional to collisionless. The higher temperatures and subsequent outflows would then arise naturally through velocity filtration by the Sun's gravitational potential [23], and may even explain the existence of the fast solar wind [14, 29].

The different properties of the low-frequency electromagnetic field and velocity fluctuations observed in the fast and slow solar wind are further evidence of the role played by turbulence and wave-particle interactions in coronal heating (Fig. 4). Fast streams contain stronger fluctuations in transverse velocity and magnetic fields, and display a higher degree of correlation between the velocity and magnetic fluctuations

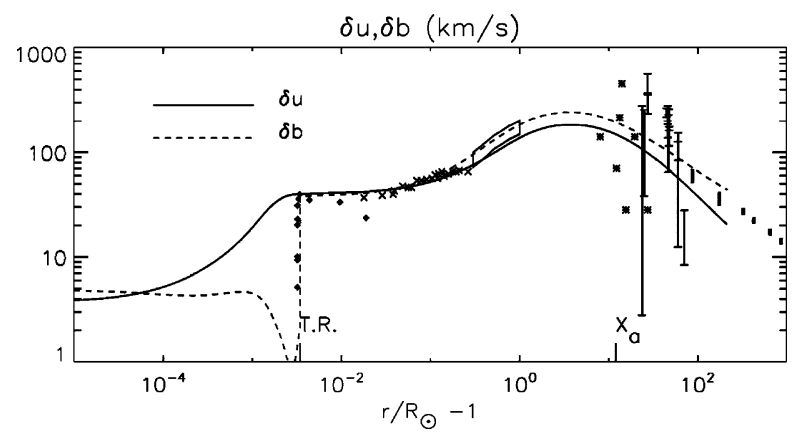

Fig. 4 The rms amplitudes $u$ and $b$ (in velocity units) as functions of heliocentric distance for a photospheric Kolmogorov spectrum with $u=40 \mathrm{~km} \mathrm{~s}^{-1}$ at the coronal base. Far symbols indicate observational constraints from in-situ measurements and inter-planetary scintillation, near symbols from remote sensing. PHOIBOS should reach inside the fluctuation maximum region, measuring spectra and correlations where the gap in the data is [27] 
(often described as a well-developed spectrum of quasi-incompressible Alfvén waves propagating away from the Sun). In the slow wind, this correlation occurs at a much lower level, while larger density and magnetic field magnitude fluctuations are present, indicating a more evolved MHD turbulent state there. This difference in turbulence state between fast and slow wind streams, together with the fact that slow wind distribution functions are much closer to equilibrium, suggests that the outward propagating wave flux contributes to the heating of the steady fast wind, while the slow wind is heated much more variably. It is not known, however, how the turbulent flux increases toward the Sun, whether it is sufficient to power coronal heating and solar wind acceleration, and how it is driven by time-dependent events in the photosphere, chromosphere, transition region, and low corona.

Whether the solar corona is heated by low-frequency waves resulting from motions naturally arising in the photosphere or whether the dominant energy source resides in the currents stored via slower field line motions has been the subject of strong debate. Among the MHD waves, only Alfvén waves would appear to survive the strong gradients in the chromosphere and transition region, because slow modes steepen into shocks while fast modes suffer total reflection. Transmitted waves propagate at large angles to the radial direction, due to the large Alfvén speed, low frequencies and strong structuring of the corona [26]. Waves reaching the lower corona should therefore be shear Alfvén waves, although discrete coronal structures such as loops and plumes might channel surface waves and propagate energy as global oscillations as well.

Simulations show that, in a highly stratified atmosphere, the nonlinear interactions of Alfvén waves launched from the photosphere are able to generate and sustain an incompressible turbulent cascade, which displays the observed Alfvénicity. The efficiency of turbulence in transporting energy to the dissipative scales is, however, still unclear. The spectral slope at different coronal heights evolves with distance, subject to expansion and driving effects, affecting the radial dependence of dissipation. The initial spectrum of Alfvén waves in the photosphere cannot be constrained by in-situ data collected in the far solar wind, since local processes contribute to its shaping there [27].

Only observations close to the solar surface will help in constraining the shape of the Alfvénic spectrum with relevant implications on the role of turbulence in the acceleration of the solar wind and the heating of the corona.

By providing the first in situ measurements of the distribution functions, waves, turbulence, and electromagnetic fields from $0.3 \mathrm{AU}$ to $4 \mathrm{R}_{\mathrm{s}}$, and by correlating them with plasma and magnetic field structures, PHOIBOS will be able to answer the basic questions of how the solar corona is powered, how the energy is channeled into the kinetics of particle distribution functions in the solar corona and wind, and how such processes relate to the turbulence and wave-particle dynamics observed in the heliosphere. The comprehensive measurement of plasma and electromagnetic fluctuations in the inner solar wind $\left(<20 \mathrm{R}_{\mathrm{s}}\right)$, will determine how the energy that powers the corona and wind is dissipated and what the dominant dissipative structures are as well as the frequency spectrum of electromagnetic fluctuations. Small-scale magnetic reconnection occupies an important place in current dissipation theories of the coronal plasma. An important set of investigations on PHOIBOS will therefore be the multi-instrument detection of signatures of small-scale reconnection, 
such as bi-directional plasma jets, accelerated particles, magnetic field, and velocity gradient correlations along the trajectory. PHOIBOS's measurements of the properties of turbulence, waves, nonlinear plasma dynamics and kinetic effects in the corona and solar wind will be a watershed for all of astrophysics, where these phenomena are invoked over widely different contexts, from accretion disks to the collisionless shocks occurring in galaxy-cluster formation.

2.2 Determine magnetic field structure and dynamics in the source regions of the fast and slow solar wind

The geometry of the magnetic field expansion in the inner corona, from the photosphere out to a few solar radii, plays a fundamental role in determining density distribution and solar wind speeds in solar wind models, as the field lines define the flow tubes along which mass and energy flux are conserved. The magnetic network in the quiet Sun looks remarkably similar to the network in coronal holes in spectral lines formed at lower, transition region temperatures, while it is harder to distinguish in lines formed at $10^{6} \mathrm{~K}$. If a similar coronal heating mechanism is at work in both the quiet Sun and coronal holes, any difference in their appearance is presumably related to the magnetic field topology, including, perhaps, its time dependence. The larger densities, apparently higher electron temperature, and different chemical composition of the quiet Sun would then be the result of a larger filling factor of closed magnetic field lines compared with that in coronal holes. While the imprint of the coronal holes and of the equatorial helmet streamers in the solar wind measured in-situ is well visible in the form of fast and slow wind streams and embedded plasma sheet, the fate of the quiet Sun corona is unknown. Is the plasma in the quiet Sun confined by closed magnetic field lines, so that the fast wind is entirely of coronal hole origin? Or is there a mass loss from the quiet Sun as well, and if so, what is its speed and how does it merge with the surrounding solar wind?

The magnetic field in active regions above sunspots provides the strongest confinement of hot plasma in the corona and is seen as bright X-ray loops, which often end in cusp-like shapes at their summit. At greater heights, these develop into streamers, which at solar minimum are large and elongated and form a belt around the solar magnetic equator. Remote sensing observations by SOHO/UVCS of the EUV emission lines of minor ions, combined with multi-fluid models [19], provide some clues about the source regions of the slow solar wind in coronal streamers, but the magnetic field topology in these regions and the role it plays in plasma outflow are unknown.

The complexity of the magnetic field structure increases with increasing activity during the solar cycle. At activity maximum, disk observations show the existence of very complicated loop structures, and images of the extended corona show streamers protruding from the solar surface not only in the equatorial regions but at all latitudes around the disk as well. PHOIBOS will determine where the slow solar wind forms in and around streamers and whether specific magnetic signatures, such as embedded current-sheets, are associated with its formation. Further, studies of solar wind sources during periods of solar maximum indicate a contribution to the wind from inside active regions as well. PHOIBOS will determine the topology of magnetic field lines within active regions that give rise to solar wind flow. 
PHOIBOS will travel over coronal holes, the quiet Sun, and the active solar corona at distances between 9 and $4 \mathrm{R}_{\mathrm{s}}$ and under both solar minimum and maximum conditions. It will trace the origin of the fast and slow wind and correlate the flow speed with closed/open magnetic field line topologies, as measured by photospheric field measurements and determined indirectly through the in-situ measurement of such parameters as electron and energetic particle bi-directional streaming. Relating the in-situ coronal observations with surface structures will require remote sensing: ecliptic viewing of the white light corona to trace field lines in the plane of the PHOIBOS orbit, tomographic images from the all-sky coronagraph to identify coronal structures in the local spacecraft environment, and a polar view of the photosphere and photospheric magnetic fields from the spacecraft perspective to identify and locate the source region structures.

The LASCO and UVCS telescopes on the SOHO mission have made important contributions to our knowledge of the origins of the slow solar wind streams around helmet streamers. Sequences of LASCO difference images obtained in 1996 (sunspot minimum) give the impression of a quasi-continuous outflow of material in "puffs" from the streamer belt [24]. A quantitative analysis of moving features shows that they originate above the cusp of helmet streamers and move radially outward, with a typical speed of $150 \mathrm{~km} / \mathrm{s}$ near $5 \mathrm{R}_{\mathrm{s}}$, increasing to $300 \mathrm{~km} / \mathrm{s}$ at $25 \mathrm{R}_{\mathrm{s}}$. The average speed profile is consistent with an isothermal corona at the temperature $T \approx 1.1 \times 10^{6} \mathrm{~K}$ (UVCS/SOHO measurements indicating a temperature $1.6 \times 10^{6} \mathrm{~K}$ in the streamer core, at activity minimum) and a critical point near $5 \mathrm{R}_{\mathrm{s}}$. The ejection of material may be caused by loss of confinement due to pressure-driven instabilities as the heated plasma accumulates or to current-driven instabilities (tearing and or kinktype instabilities) in the sheared field of the streamer. PHOIBOS will cross the paths of these ejecta from streamers and will ascertain whether the ejection of coronal material occurs in a continuous flow or whether the puffs are in fact disconnecting plasmoids. If the latter, PHOIBOS will determine the magnetic field configuration of the plasmoid as well as the magnetic morphology at the point of disconnection in the corona. Comparison of Galileo radio data with UVCS/SOHO images clearly shows the association of the slow wind with streamer stalks, that is, with the regions above the cusps of helmet streamers that include the current sheet [11]. It is not known, however, whether there is a single current sheet that runs along the nearly equatorial strip of maximum brightness in the white corona, i.e., along the streamer belt (as surmised by [28]), or whether there are a number of stalk/sheet structures of finite longitudinal extent. Nor is the structure of current sheets in streamer stalks known. Do they have a simple structure, or are they made up of multiple sheets in a more complex magnetic field morphology, as is suggested in part by UVCS/SOHO measurements [18] and multiple current sheet crossings in-situ [25].

As observed in-situ at large distances from the Sun, the solar wind appears as a continuous plasma outflow. Its quasi-steady character may be a property of the outflow at the solar source. However, the apparently quasi-stationary wind may also result from a number of spatially limited, impulsive events that are distributed over smaller scales [6]. There is abundant evidence for the intermittent or "pulsed" [6] character of the high-speed wind: observations of microstreams and persistent beamlike features in the fast wind; interplanetary scintillation measurements of fieldaligned density structures having a 10:1 radially-aligned axial ratio and apparent 
field-aligned speeds ranging from $\sim 400$ to $\sim 1,280 \mathrm{~km} / \mathrm{s}[2,9]$; and remote sensing observations of the chromosphere, transition region, and corona revealing explosive, bursty phenomena, dubbed microflares, associated with magnetic activity over an extremely wide range of energy and time scales. Feldman et al. [5, 6] have interpreted the fine-scale structures observed in the fast wind as remnants of spicules, macrospicules, X-ray jets, and $\mathrm{H}$-alpha surges and hypothesize that the fast wind results from the superposition of transient reconnection-generated jets. If this hypothesis is correct, then the heating of the corona leading to its time-dependent acceleration to form an ensemble of outward-going jets could be accompanied by the annihilation of oppositely-directed magnetic flux bundles clustered near the magnetic network, in turn leading to transient hard X-ray and gamma-ray bursts, along with neutron production in the 1 to $10 \mathrm{MeV}$ energy range, which could be detected by PHOIBOS.

For the slow solar wind, evidence in favor of an intermittent origin is even more abundant. As mentioned above, blobs of plasma appear to be lost by helmet streamer structures overlying active regions and various mechanisms have been proposed for this process. At solar maximum, an important and definitely intermittent solar wind component is present in the form of CMEs and the fine-scale structure of the solar wind from active regions supports at least a spatially structured origin for the various flow streams. More generally, smaller CME-like events at all scales could contribute significantly to the solar wind throughout the activity cycle.

Direct in-situ measurements of the structure of the solar wind, of the ion and electron distribution functions, as well as elemental abundance variations close to the Sun are required to understand the source regions of the wind. PHOIBOS will directly measure both the electron distribution function and flow speeds of minor ions in the coronal hole, and, at perihelion, may directly sample composition differences on closed and open fields. By continuous direct sampling the plasma flow as it moves close to the Sun, PHOIBOS will be able to assess the space and time filling factor of the fast solar wind, while imaging the coronal structures that it will cross in the range above $10 \mathrm{R}_{\mathrm{s}}$. The time-dependent variability observed in the wind might also increase close to the Sun, leading to effects of multiple sources observable by PHOIBOS, for example from a multitude of bursty events or micro CME's.

To locate the source regions of the solar wind, in-situ ion, plasma and magnetic field measurements at high resolution are necessary covering an extended field of view due to the contribution to aberration of Alfvénic turbulence (maximum estimated to be $200 \mathrm{~km} / \mathrm{s}$, or $\Delta u / U=0.3$ ), together with images both of the underlying photospheric field and the quasi-simultaneous coronograph all sky images as well as context from telescopes in earth orbit. Bi-directional electron streaming could identify position on closed/open field lines. Note that for fast particles, the aberration is essentially due to $\mathrm{db} / B$ (maximum estimated in the range $0.2-0.3)$. In coronal holes, filamentary structures such as coronal plumes are observed out to $30 \mathrm{R}_{\mathrm{s}}$, the range of speeds between $300 \mathrm{~km} / \mathrm{s}$ (perihelion) implies speeds across plumes in the range 100-200 km/s. With an expected size less than $10^{3}-10^{5} \mathrm{~km}$ at $8 \mathrm{R}_{\mathrm{s}}$, crossing time of an individual plume should last between $5 \mathrm{~s}$ to $1 \mathrm{~h}$. During that time one must ensure a sufficient number of plasma, particle and velocity measurements. Radio measurements at the plasma frequency appear also 
essential to give a separate and independent measure of density, speed and temperature of the core of the electron distribution function.

2.3 What mechanisms accelerate, store, and transport energetic particles?

Two kinds of solar energetic particle (SEP) events occur during active periods, often both together: gradual events, in which particles are accelerated in the corona by shocks driven by fast coronal mass ejections (CMEs), and impulsive events, in which particles are accelerated by solar flares. In addition, even at the quietest times there is a continuous outflow from the Sun of particles of intermediate energies (suprathermal to $>10 \mathrm{MeV}$; Fig. 5). The mechanism responsible for this outflowmicroflares? - is not known. Further questions concern the relative contributions of reconnection, shocks, and turbulence to particle acceleration in impulsive events, the identity and source of seed populations for gradual events, and the means by which energetic particles are transported to high latitudes. Accomplishment of this objective will not only advance our understanding of a fundamental plasma process, energetic particle acceleration, but will also significantly contribute to efforts to predict SEPs, which present one of the most serious threats to astronaut health and safety.

- $\quad$ PHOIBOS will measure the solar wind and magnetic field close to the Sun, the density and energy spectrum of suprathermal seed particles, and the spectrum of magnetic turbulence directly. It will thus be able to ascertain the presence of shocks and discontinuities and determine their role in particle acceleration.

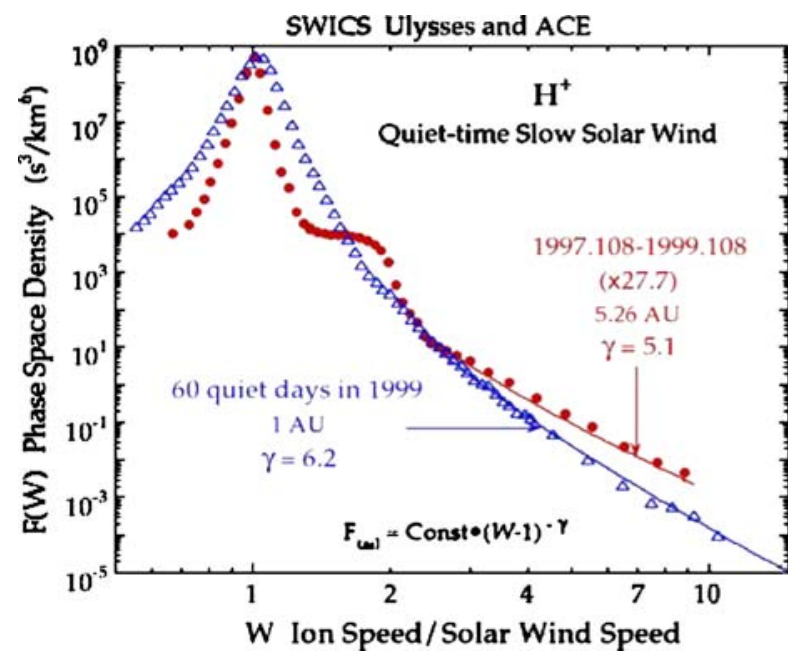

Fig. 5 All solar wind species that have been measured develop suprathermal tails extending to $>100 \mathrm{keV} / \mathrm{nuc}$ somewhere between the Sun and 1 AU [8]. These tails are continuously present, even in the absence of solar activity or interplanetary shocks. The mechanism responsible for creating these tails is not known. Solar Probe will investigate possible acceleration processes by exploring the inner heliosphere to distances where no spacecraft has ventured before 


\subsection{Dust in the inner heliosphere}

The inner heliosphere is populated with dust grains originating from comets and asteroids. This inner heliospheric dust cloud, the source of the zodiacal light and the Sun's F-corona, has not been well characterized. PHOIBOS's unique path near the Sun will make it possible to answer questions about the size and mass distribution of the dust, about its composition and origin, and about its interaction with the near-Sun plasma and gas environment. Of particular interest is the contribution of the dust to the "inner source" of energetic particles. As its fourth objective, PHOIBOS will explore dusty plasma phenomena in the near-Sun environment and their influence on the solar wind and energetic particle formation.

\section{PHOIBOS science implementation}

PHOIBOS will address the science objectives through a combination of in-situ and remote-sensing observations performed from at the Sun. Inside a distance of $0.3 \mathrm{AU}$ on both sides of perihelion, PHOIBOS will make in-situ measurements of plasma, supra-thermals, energetic particles, magnetic fields, waves, and dust in the near-Sun environment. EUV and magnetic imaging of solar wind source regions and whitelight imaging of coronal structures will be performed on both the inbound and outbound legs of the solar pass. The remote-sensing observations will allow in-situ measurements to be related to magnetic and plasma structures at the Sun. Closest approach will occur at a perihelion altitude of $3 \mathrm{R}_{\mathrm{s}}$ above the surface. Supporting remote-sensing observations from ground-based, sub-orbital, and space based assets will be coordinated with perihelion passes to provide context for Solar Probe's insitu measurements. A dedicated theory and modeling program will be an integral part of the PHOIBOS mission.

The PHOIBOS spacecraft will use an Ariane AR5 ECA launcher and a plasmic propulsion stage in order to reach it's final scientific orbit described in Table 1 . The required escape conditions are a modulus of the hyperbolic excess velocity $v_{\infty}=$ $3.0 \mathrm{~km} / \mathrm{s}$ and a declination of the hyperbolic excess velocity $\delta_{\infty}=-6 \mathrm{deg}$. The launcher performance for these conditions is $5,000 \mathrm{~kg}$ offering $25 \%$ of margin.

The overall mission profile is summarized in Figs. 6 and 7. For a launch in December 2018, the acquisition of the final orbit is planned in June 2027, the first flyby of the Sun at $4 \mathrm{R}_{\mathrm{s}}$ will take place on 29 September 2027 and the second one on 29 January 2030 (Table 1). The launch opportunity for such a scenario is a window every 584 days (1.6 years). The earlier possible launch dates are therefore May 2017

Table 1 PHOIBOS final orbit characteristics

\begin{tabular}{ll}
\hline Characteristics & Values \\
\hline Perihelion radius & $4 \mathrm{R}_{\mathrm{s}}$ \\
Aphelion radius & $3.5 \mathrm{AU}$ \\
Sidereal period & 853 days \\
& $(2.33$ years $)$ \\
Eccentricity & $e=0.9886$ \\
Inclination with respect to the ecliptic plane & $60^{\circ}$ \\
& \\
\hline
\end{tabular}




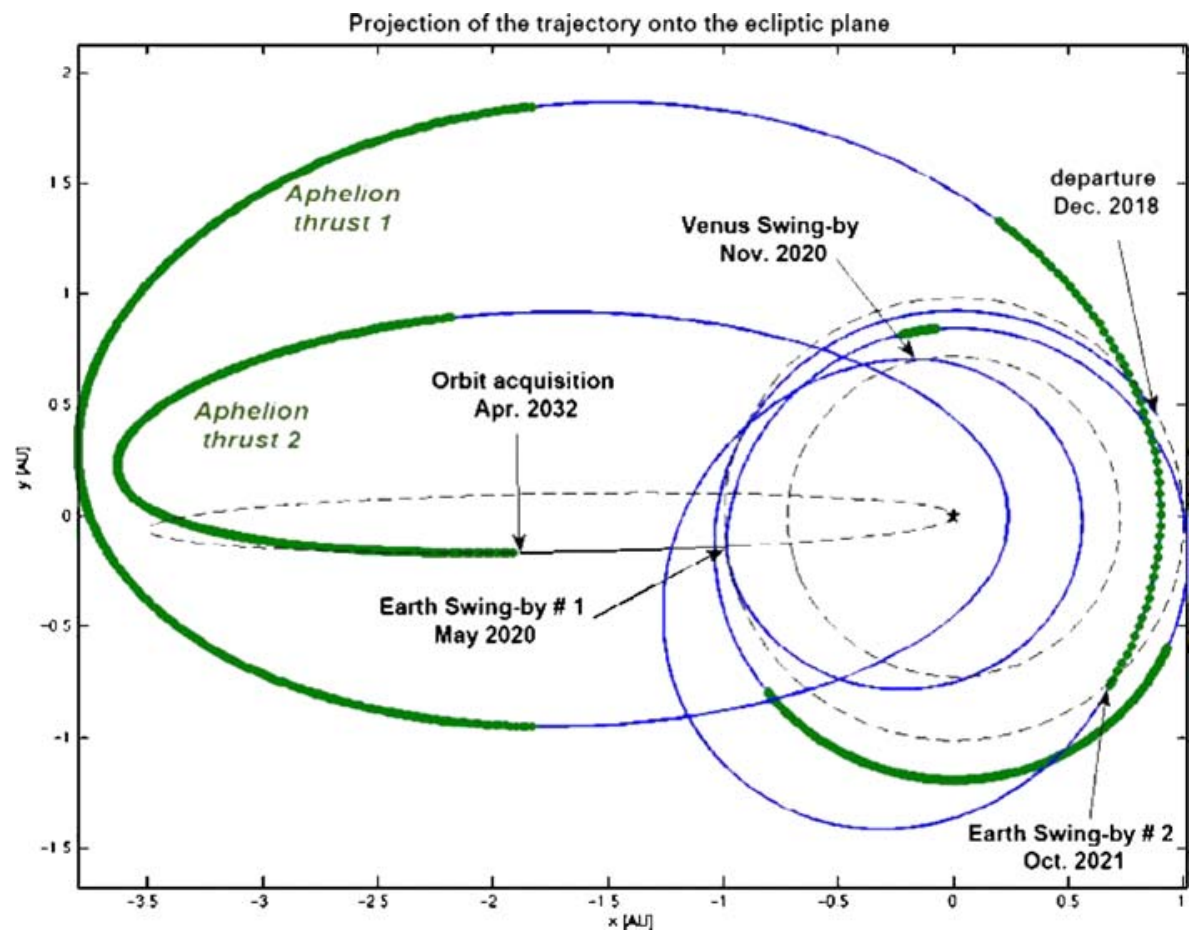

Fig. 6 Projection of the PHOIBOS trajectory on the ecliptic plane. The plasma thrusting phases are indicated in green
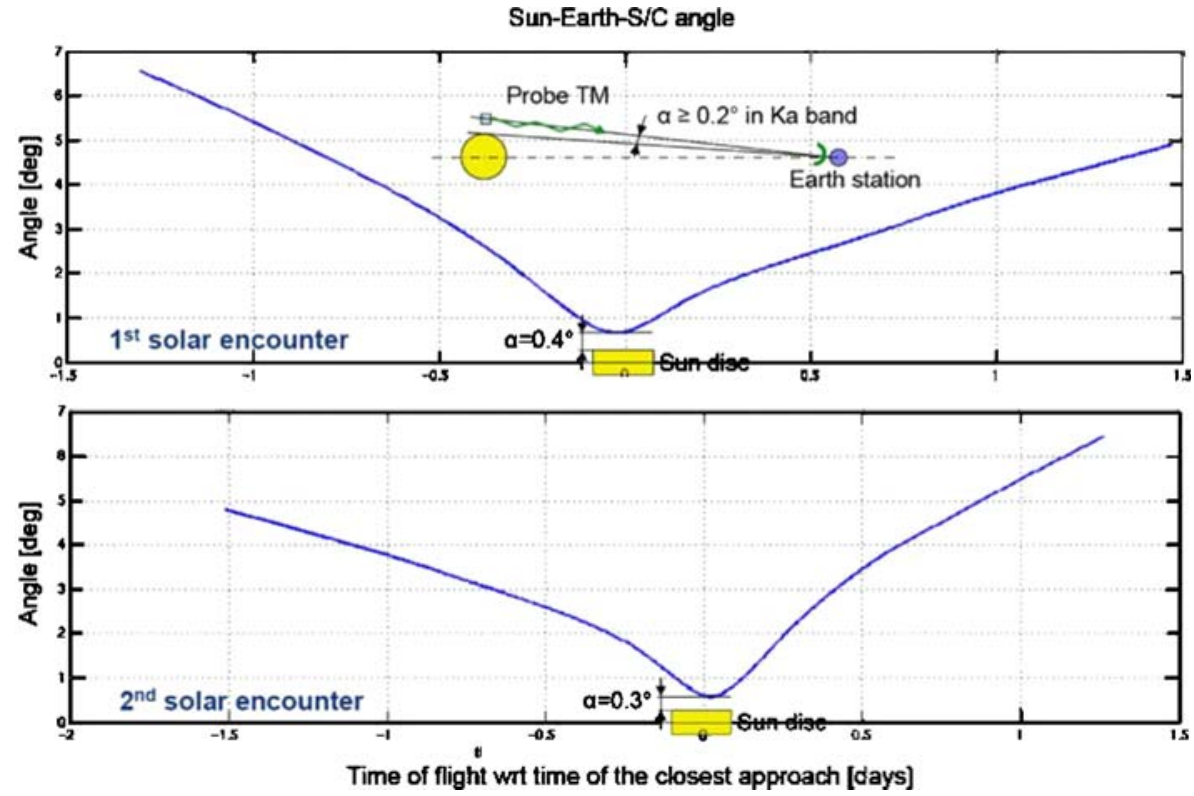

Fig. 7 Sun-Earth-S/C angle as a function of time for the first two solar encounters 
or October 2015. The final launch date should also be chosen so that the first flyby of the Sun occurs during solar minimum of activity which is approximately the case for a December 2018 launch. In order to reach the final orbit, a total Delta-V of 12.25 $\mathrm{km} / \mathrm{s}$ is necessary. To obtain such a Delta-V, two Earth swing-by (March 2025 and August 2026), one Venus swing-by (September 2025) and plasmic propulsion are necessary. The envisaged plasmic engines are PPS5000 with one or two engines working in parallel depending on the available power (specific impulse, Isp $=1900 \mathrm{~s}$, thrust magnitude, $185 \mathrm{mN}<F<280 \mathrm{mN}$, electrical power, $3 \mathrm{~kW}<P<4.5 \mathrm{~kW}$ ). The total duration of the cruise phase will be 8.5 years (3,100 days).

The Sun closest approaches during the cruise phase will be in August 2025 (0.56 AU) and December 2029 (0.26 AU), which is equivalent to the ESA Solar Orbiter mission. The two most efficient thrusting phases in term of Delta-V will occur at heliocentric distances between 1.8 and 3.7 AU. These phases are indicated on Fig. d. 1 as aphelion thrusts 1 and 2. While the main thrusting axis during the "aphelion thrust 1" period remains in the ecliptic, for "aphelion thrust 2" the probe will be oriented in such a way, that thrusting will allow not only to reach the necessary Delta-V in order to lower the perihelion, but also to increase the inclination of the final orbit up to $60^{\circ}$ from the ecliptic plane. The total Xenon mass for the plasmic engines required for the mission is $1,949 \mathrm{~kg}$. Therefore the final spacecraft composite dry mass will be around $2,050 \mathrm{~kg}$.

A slight variation to the proposed scenario with only one Earth swing-by and a total duration for the cruise phase of 7.5 years could be envisaged with the use of the new generation of Ariane 5 launcher (Ariane 5 ECB). Finally, one should note also that the use of the PROTON M launcher, with its BREEZE M upper stage fulfils also, albeit without performance margin, the mission profile that we have described.

Since the most valuable scientific measurements are performed during the Sun's closest approaches, the tracking and command capabilities are mainly driven by the ability to download in real time as most of the data as possible Fig. 3.2 displays the Sun-Earth-S/C configurations during the first two solar passes. X-band/Ka-band downlink allows (1) to gain $11.6 \mathrm{~dB}$ of $S / N$ ratio wrt the coronal scintillation and (2) a beam of $0.2^{\circ}$ allowing a Earth antenna tracking at $0.2^{\circ}$ from the Sun disc.

A BepiColombo configuration allows a telemetry of $45 \mathrm{kbits} / \mathrm{s}$ at $1 \mathrm{AU}$. However, taken into account the $35 \mathrm{~m}$ Earth antenna temperature noise when turned toward the Sun, a flow of $2 \mathrm{kbits} / \mathrm{s}$ can be expected for the TM budget at the most critical point. It will be necessary therefore to use a large onboard memory to store as much data as possible during the solar passes and process and transmit them after perihelion.

The complete on board communication system is based on the Bepi Colombo one. Its features can be estimated to $25 \mathrm{~kg}$ in mass and $90 \mathrm{~W}$ in consumption.

\section{Payload description}

The overall philosophy of the current proposal in terms of scientific objectives and necessary payload in order to achieve them has greatly been inspired by previous Solar Probe proposals and especially by the last NASA STDT/2005 report.

To meet the PHOIBOS science objectives, a payload comprising both comprehensive in-situ and relevant remote-sensing instruments was selected. 
However, the in-situ payload should have the priority in terms of resources and accommodation trade-offs. The complementary remote sensing observations may be provided by Solar Orbiter, depending upon the schedules of the two missions.

PHOIBOS in-situ instrumentation consists of a Fast Ion Analyzer, a Fast Electron Analyzers, an Ion Composition Analyzer, an Energetic Particle Instrument, a Magnetometer, a Radio and Plasma Wave Instrument, a Neutron/Gamma Ray Spectrometer, and a Coronal Dust Detector. The remote-sensing instrumentation comprises a Hemispheric Imager for white-light imaging of coronal structures and a Polar Source Region Imager for EUV and magnetic imaging of the photosphere. The payload is serviced by a common data processing unit (CDPU) and low voltage power supply (LVPS). Table 2 summarizes the contribution of the various instruments to the PHOIBOS scientific objectives. The mass, power, and data rate allocations for the baseline payload are shown in Table 3. They are based on those of past or existing instrumentation or components.

\section{Spacecraft description}

The spacecraft is constituted by the Phoibos Transfer Module (PTM) and by the Phoibos Solar Probe (PSP), both integrated into the Phoibos Composite Spacecraft (PCS) at launch and during transfer. The mission will commence in 2023 with the launch of the PCS on an Ariane 5. After a long interplanetary cruise phase, during which the PCS is powered by the transfer module (PTM), the probe (PSP) will be delivered to its final solar high elliptical orbit in 2032.

Table 2 Science objectives and contributing instruments

\begin{tabular}{|c|c|c|c|c|c|c|c|c|c|c|}
\hline Scientific objectives & FIA & FEA & ICA & EPI & RPWI & MAG & NGS & $\mathrm{CD}$ & PSRI & $\mathrm{HI}$ \\
\hline $\begin{array}{l}\text { Explore the fundamental processes } \\
\text { underlying coronal heating } \\
\text { and solar wind acceleration }\end{array}$ & $\mathrm{R}$ & $\mathrm{R}$ & $\mathrm{R}$ & $\mathrm{S}$ & $\mathrm{R}$ & $\mathrm{R}$ & $\mathrm{S}$ & & $\mathrm{S}$ & $\mathrm{S}$ \\
\hline $\begin{array}{l}\text { Determine magnetic field structure and } \\
\text { dynamics in the source regions of the } \\
\text { fast and slow solar wind }\end{array}$ & $\mathrm{R}$ & $\mathrm{R}$ & $\mathrm{R}$ & $\mathrm{S}$ & $\mathrm{S}$ & $\mathrm{R}$ & $\mathrm{S}$ & & $\mathrm{R}$ & $\mathrm{R}$ \\
\hline $\begin{array}{l}\text { Determine what mechanisms store, } \\
\text { accelerate and transport energetic } \\
\text { particles }\end{array}$ & $\mathrm{R}$ & $\mathrm{R}$ & $\mathrm{R}$ & $\mathrm{R}$ & $\mathrm{R}$ & $\mathrm{R}$ & $\mathrm{S}$ & & $\mathrm{S}$ & $\mathrm{S}$ \\
\hline $\begin{array}{l}\text { Explore dust and dusty plasma } \\
\text { phenomena } \\
\text { in the vicinity of the Sun and their } \\
\text { influence on the solar wind and } \\
\text { energetic particle formation }\end{array}$ & $\mathrm{R}$ & $\mathrm{R}$ & $\mathrm{R}$ & $\mathrm{S}$ & $\mathrm{S}$ & $\mathrm{S}$ & & $\mathrm{R}$ & & $\mathrm{S}$ \\
\hline
\end{tabular}

FIA Fast ion analyzer; FEA fast electron analyzer; ICA ion composition analyzer; EPI energetic particle instrument; $M A G$ DC magnetometer; $R P W I$ radio and plasma waves instrument; $N G S$ neutron/gamma-ray 
Table 3 Instrument resource requirements

\begin{tabular}{lccc}
\hline Instrument & Mass (kg) & Power (W) & Peak data rate (kbps) \\
\hline Fast ion analyzer (FIA) & 2.5 & 3.7 & 10 \\
Fast electron analyzer (FEA) & 5.0 & 7.2 & 20 \\
Ion composition analyzer (ICA) & 6.0 & 6.0 & 10 \\
Magnetometer (MAG) & 1.5 & 2.5 & 1.1 \\
Radio and plasma wave instrument (RPWI) & 7.0 & 5.0 & 4 \\
Energetic particle instrument, low energy (EPI-Lo) & 1.5 & 2.3 & 5 \\
Energetic particle instrument, high energy (EPI-Hi) & 2.5 & 1.7 & 3 \\
Neutron/gamma ray spectrometer (NGS) & 2.5 & 3.0 & 0.5 \\
Coronal dust detector (CD) & 1.5 & 3.8 & 0.1 \\
Hemispheric imager (HI) & 1.5 & 4.0 & 70 \\
Polar source region imager (PSRI) & 3.5 & 4.0 & 70 \\
Common DPU/LVPS & 10 & 14 & N/A \\
Total & 45.0 & 57.2 & 123.7 \\
\hline
\end{tabular}

Source-NASA STDT 2005 Report

\subsection{Spacecraft architecture}

\subsubsection{Composite spacecraft architecture}

The Phoibos Composite Spacecraft (PCS) is composed of the Solar probe, mounted on top of the transfer module, featuring four solar arrays of Rosetta size, mounted by pair on two Solar Array Drive Mechanisms (SADM), and five or six plasmic engines (see discussion below). Figure 8 shows the PCS in launch configuration.

After the launch phase and the early operations, the solar arrays are deployed and oriented so that they are perpendicular to the orbit, facing the Sun. The spacecraft

Fig. 8 Phoibos Composite Spacecraft in launch configuration
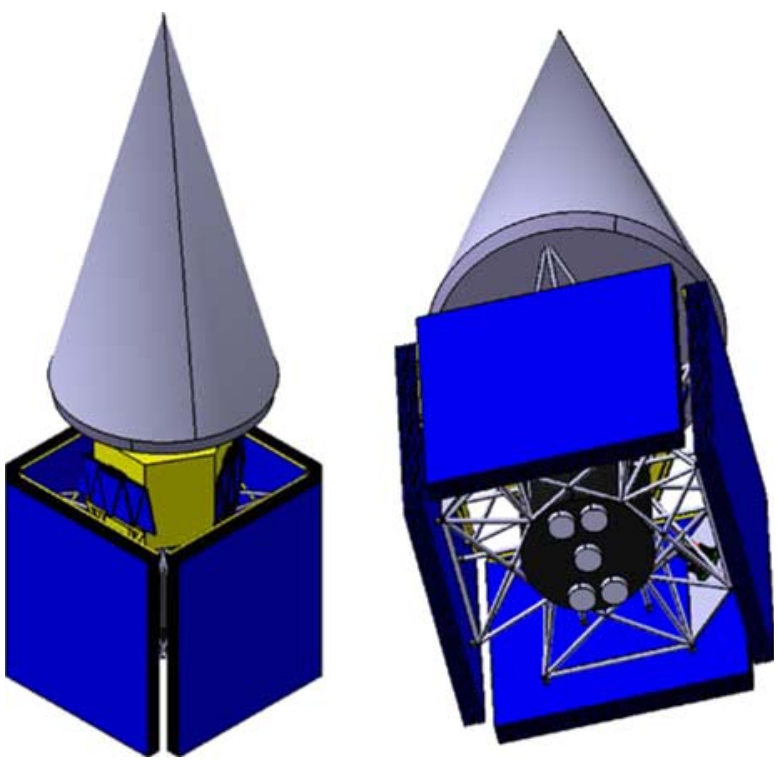
body lies therefore in the orbit plane and can rotate freely thanks to the SADM around the orbit normal direction to orient the plasmic engines during propulsive arcs. Figure 9 shows the PCS in deployed configuration during the cruise phase. The control of the PCS is ensured by the Probe data management system and AOCS sensors, but using dedicated actuators (wheels and hydrazine system). The solar panels are orientated with null incidence with respect to Sun for the approach at 0.26 AU during the cruise (after the second propulsive arc), and a dedicated protection system (small heat shield on the side of each panel) will be accommodated. There is no propulsion required below $0.85 \mathrm{AU}$.

\subsubsection{Phoibos solar probe architecture}

The Phoibos Solar Probe (PSP) spacecraft is composed of a conical Heat Shield and of an orbiter including the science payloads and the platform. The PSP is separated from the transfer module as soon as the science target orbit is reached (Fig. 10).

The probe includes three solar panels of $1.36 \mathrm{~m}^{2}$ each that can be folded behind the heat shield during perihelion passage, using the one axis orientation mechanism on each panel.

\subsubsection{The heat shield}

The heat shield is based on the strong Solar Orbiter heritage, and from the NASA STDT/2005 study. Promising related tests are also currently being carried out at the Odeillo Solar furnace facility in France. These tests are intended to study $\mathrm{C} / \mathrm{C}$ composites behaviours under high temperature, ion and UV irradiations $[4,21]$.

The heat shield has a conical shape with a $15^{\circ}$ half-cone angle and is built with $\mathrm{C} /$ $\mathrm{C}$ structure. Depending on the acceptable conduction in the struts supporting the shield, basically two options are possible regarding the coating of the shield. A white coating will result in an $\alpha / \varepsilon$ around 0.6 and a cooler shield. Bare $\mathrm{C} / \mathrm{C}$ will result in an $\alpha / \varepsilon$ around 1 and a hotter shield. For instance, the thermal load received by the heat shield at perihelion being around $21 \mathrm{MW}$, the heat shield external layer temperature will be around $1,800^{\circ} \mathrm{C}$ in the case of white coating. Note however that two problems may occur with the white coating. Firstly the value of $\alpha / \varepsilon$ around 0.6 should be measured at higher temperatures in order to check whether it is constant or no. Secondly, the problem with the white coating is that possible cracks can occur and also delamination due to the different coefficients of thermal expansion of the

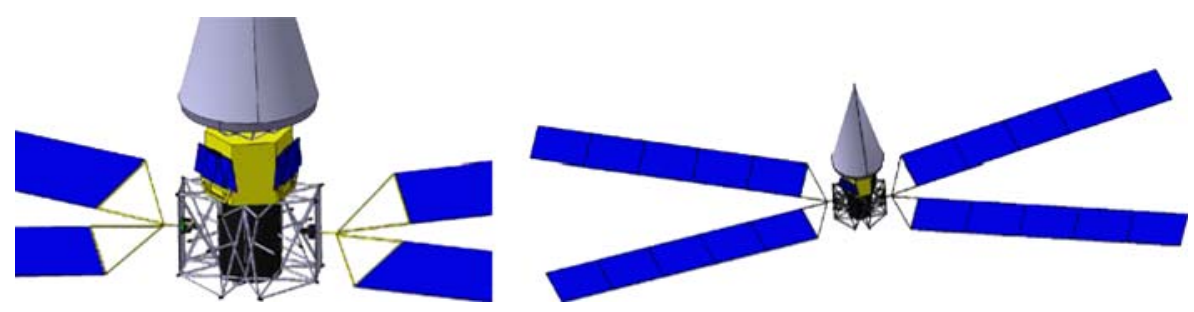

Fig. 9 Phoibos Composite Spacecraft in deployed configuration during cruise 

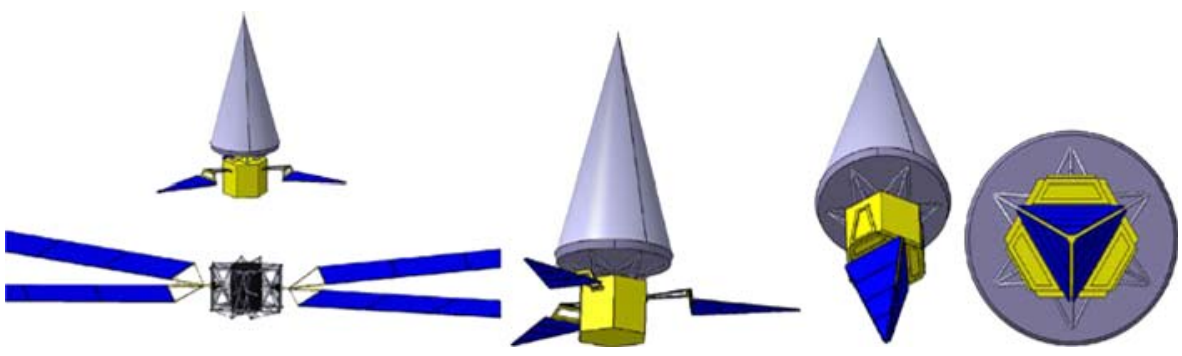

Fig. 10 Separation of the Phoibos Solar Probe from the PCS

materials. Moreover if the temperature is higher, the white coating can melt leading to very important damage of the structure.

The heat shield is separated in two parts: (1) the conical part and (2) a secondary flat heat shield that closes the cone at its base, using also Solar Orbiter technology. The total thermal input to the bus from the heat shield is around $50 \mathrm{~W}$, and the spacecraft platform is therefore kept at a temperature of $40^{\circ} \mathrm{C}$.

\subsection{Key factors for power management}

The power management is a key aspect of the Phoibos mission for both the transfer module and for the solar probe. In the composite configuration, all the power is ensured by the $130 \mathrm{~m}^{2}$ surface of solar panels (four Rosetta panels), providing the required $33 \mathrm{~kW}$ equivalent power at 1 AU. MPPT (Maximum Power Point Tracking) are used to limit currents, and the solar panels are coupled for one SADM.

\subsection{Power in the probe configuration at Aphelion and Perihelion}

In the probe configuration, the power management is ensured by the three deployable $1.36 \mathrm{~m}^{2}$ solar panels. However, these panels only provide $80 \mathrm{~W}$ at the aphelion of the science orbit. The probe is therefore put in a very low state of activity during the major part of the science orbit, with a low spin stabilization around the Sun direction, and the battery is used at regular interval to perform spacecraft control actions and communications. The battery is charged between two actions using the $80 \mathrm{~W}$ power, together with spacecraft thermal control.

From the Solar Orbiter and Bepi Colombo developments, it appears that the three panels of the probe can only be used up to $250^{\circ} \mathrm{C}$, tilted at $\sim 70^{\circ}$, for heliocentric distances down to 0.2 AU. Below this distance, the panels will therefore be stowed behind the shield and a new power system shall relay this standard one. The required power can be generated by thermo-electrical elements and converters, accommodated at the secondary heat shield level inside the cone, to take maximum benefit of the heat generated by the heat shield. A plate is used as hot source and the secondary heat shield is used as cold source with a calibrated radiator. These thermo elements have to be selected pending on the temperature range experienced by the plate. The provided power has been estimated to more than $200 \mathrm{~W}$ below $0.1 \mathrm{AU}$, but the zone between $0.2 \mathrm{AU}$ and $0.1 \mathrm{AU}$ is still a critical part of the mission: this power subsystem of the probe, working below $0.2 \mathrm{AU}$ is therefore one of the key driver of the 


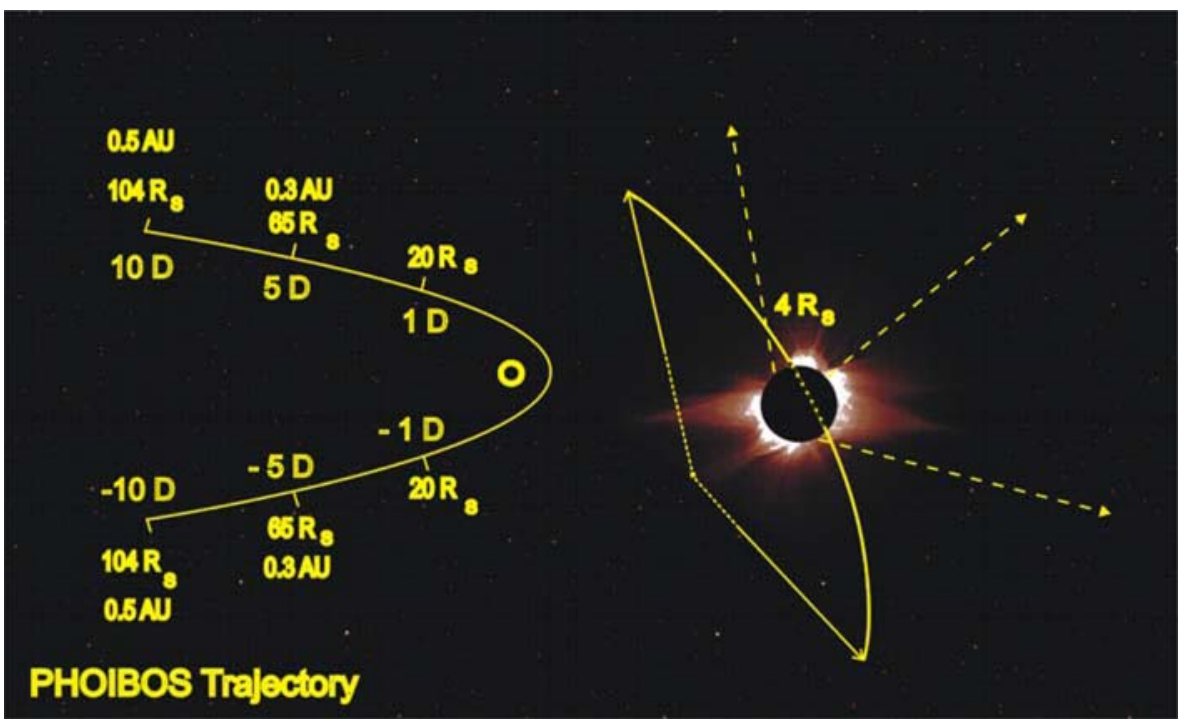

Fig. 11 PHOIBOS trajectory at closest approach showing inclination angle. Encounters begin at 0.3 AU, 5 days before closest approach, and lasts until 5 days after closest approach

probe design. An alternative to this system would be to deploy small mirrors behind the shield, reflecting part of the solar light at $4 \mathrm{R}_{\mathrm{s}}$ towards the folded solar panels. With very high emissive coating and very low absorption (that can be maintained without ageing effect since they would only be in Sun below $0.2 \mathrm{AU}$ ), a small mirror would reach $\sim 1,200 \mathrm{~K}$, below its fusion temperature.

\section{Conclusions}

PHOIBOS' perihelion passage - the first spacecraft to fly within four stellar radii of a star-will be such a unique, exciting, and important scientific opportunity for the solar, space physics and astro-physics communities that all relevant national and international scientific assets should be dedicated to providing supporting observations in a coordinated perihelion pass campaign. The solar encounter itself will be treated as a climactic event, analogous to planetary conjunctions or comet approaches to the Sun. Indeed, PHOIBOS is a man-made Sun-grazing comet.

PHOIBOS will be an exciting mission of exploration, discovery, and deep understanding. It will journey to one of the last unexplored regions of the solar system and reveal how the corona is heated and the solar wind accelerated, solving two fundamental mysteries.

Acknowledgements $M$. Velli would like to thank D. McComas and W. Lewis for many useful discussions and E. Podlachikova for providing Fig. 11. M Velli was partially supported by NASA SEC Advanced Studies. Milan Maksimovic would like to thank the CNES since the overall mission profile presented here is the outcome of a specific CNES/PASO study conducted by Régis Bertrand for the trajectory, Emmanuel Hinglais for the system analysis and Jean-Yves Prado for the overall concept. This study has been performed in the frame of the CNES support to the French scientific contributors to the 
ESA "Cosmic Vision" call. The spacecraft accommodation has been provided by EADS/Astrium. Finally the authors would like to thank the PHOIBOS scientific team composed by Thierry Appourchaux, Bruno Bavassano, Stuart D. Bale, Matthieu Berthomier, Douglas Biesecker, Lars Blomberg, Peter Bochsler, Volker Bothmer, Jean-Louis Bougeret, Andrew Breen, Carine Briand, Roberto Bruno, Vincenzo Carbone, Patrick Canu, Thomas Chust, Jean-Marc Defise, Thierry Dudok de Wit, Luca Del Zanna, Anders Eriksson, Silvano Fineschi, Lyndsay Fletcher, Keith Goetz, Roland Grappin, Antonella Greco, Shadia Habbal, Don Hassler, Bernd Heber, Petr Hellinger, Tim Horbury, Karine Issautier, Justin Kasper, Ludwig Klein, Craig Kletzing, Säm Krucker, Vladimir Krasnoselskikh, William Kurth, Rosine Lallement, Philippe Lamy, Hervé Lamy, Simone Landi, Olivier Le Contel, Fabio Lepreti, Dominique LeQuéau, Robert Lin, Milan Maksimovic, Francesco Malara, Ian Mann, Ingrid Mann, William Matthaeus, Dave McComas, Ralph McNutt, Nicole Meyer-Vernet, Zoran Mikic, Michel Moncuquet, Neil Murphy, Zdenek Nemecek, Emanuele Pace, Filippo Pantellini, Viviane Pierrard, Jean-Louis Pinçon, Elena Podlachikova, Raymond Pottelette, Lubomir Prech, Ondrej Santolik, Robert Rankin, Franco Rappazzo, Marco Romoli, Alain Roux, Jana Safrankova, Fouad Sahraoui, Edward C. Sittler, Luca Sorriso-Valvo, Jan Soucek, Pavel Travnicek, Andris Vaivads, Andrea Verdini, Nicole Vilmer, Robert Wimmer-Schweingruber, Gaetano Zimbardo, Thomas Zurbuchen, Ioannis Zouganelis.

\section{References}

1. Antonucci, E., et al.: Spectroscopic measurement of the plasma electron density and outflow velocity in a polar coronal hole, Astron. Astrophys., 416, 749 (2004)

2. Coles, W.A., et al.: Comparison of solar wind velocity measurements with a theoretical acceleration model, J. Geophys. Res. 96, (13), 849 (1991)

3. David, C., et al.: Measurement of the electron temperature gradient in a solar coronal hole, Astron. Astrophys. 336, L90 (1998)

4. Eck, J.: PhD thesis in preparation (2007)

5. Feldman, W.C., et al.: Constraints on high-speed solar wind structure near its coronal base: Astron. Astrophys. 316, 355 (1996)

6. Feldman, W.C., et al.: Experimental constraints on pulsed and steady state models of the solar wind near the Sun, J. Geophys. Res. 102, (26), 905 (1997)

7. Gabriel, A.H., et al.: Solar Wind Outflow in Polar Plumes from 1.05 to 2.4 Rsolar, Astrophys. J., 635, L185-L188. (2005)

8. Gloeckler, G., et al.: Sources, injection, and acceleration of heliospheric ion populations, In: Mewaldt, R.A., et al. (eds.) AIP Conference Proceedings 528, 221 (2000)

9. Grall, R.R., et al.: Rapid acceleration of the polar solar wind, Nature 379, 429 (1996)

10. Grappin, R., Mangeney, A., Marsch, E.: On the origin of solar wind MHD turbulence: HELIOS data revisited, J. Geophys. Res. 95, 8197 (1990)

11. Habbal, S.R., et al.: Origins of the slow and the ubiquitous fast solar wind, Astrophys. J. 489, L103 (1997)

12. Kohl, J.L., et al.: UVCS/SOHO empirical determinations of the anisotropic velocity distributions in the solar corona, Astrophys. J. 501, L127-L131 (1998)

13. Li, X., et al.: The effect of temperature anisotropy on obsevations of Doppler dimming and pumping in the inner corona, Astrophys. J. 501, L133 (1998)

14. Maksimovic, M., et al:: A kinetic model of the solar wind with Kappa distribution functions in the corona, Astron. Astrophys. 324, 725 (1997)

15. Maksimovic, M., Velli, M.: The Phoibos mission: probing heliosphereic origins with an inner boundary observing spacecraft, a proposal to ESA for a probe of the Solar Corona and inner Heliosphere in response to the call for "Cosmic Vision" (2007)

16. McComas, D.J., et al.: The three-dimensional solar wind around solar maximum, Geophys. Res. Lett. 30, 24-1 (2003). doi:10.1029/2003GL017136

17. McComas, D.J., et al.: Solar probe science and technology definition team report, NASA (2005)

18. Noci, G., et al.: First results from UVCS/SOHO, Adv. Space Res., 20, 2219 (1997)

19. Ofman, L.: Three-fluid model of the heating and acceleration of the fast solar wind, J. Geophys. Res. 109, A07102 (2004). doi:10.1029/2003JA010221

20. Ofman, L.: MHD waves and heating in coronal holes, Space Sci. Rev. 120, (1-2), 67 (2005). doi:10.1007/s11214-005-5098-1 
21. Paulmier, T., et al.: Physico-chemical behavior of carbon materials under high temperature and ion irradiation, Appl. Surf. Sci. 180, 227 (2001)

22. Rosenbauer, H., et al.: A survey on initial results of the HELIOS plasma experiment, J. Geophys. 42, 561 (1977)

23. Scudder, J.D.: Ion and electron suprathermal tail strengths in the transition region: Support for the velocity filtration model of the corona, Astrophys. J. 427, 446 (1994)

24. Sheeley Jr., N.R., et al.: Measurements of Flow Speeds in the Corona between 2 and 30 Rs, Astrophys. J. 484, 472 (1997)

25. Smith, E.J.: The heliospheric current sheet, J. Geophys. Res. 106, (15), 819 (2001)

26. Velli, M.: On the propagation of ideal, linear Alfve'n waves in radially stratified stellar atmospheres and winds, Astron. Astrophys. 270, 304 (1993)

27. Verdini, A., Velli M.: Alfvén Waves and Turbulence in the Solar Atmosphere and Solar Wind, Astrophys. J. 662, 669 (2007)

28. Wang, Y.-M., et al.: Solar wind stream interactions and thewind speed-expansion factor relationship, Astrophys. J. 488, L51 (1997)

29. Zouganelis, I., et al.: A transonic collisionless model of the solar wind, Astrophys J. 606, 542 (2004) 